\title{
Efficient Sideband Noise Cancellation for Co-located Networks Using ANCT
}

\section{Nosiri Onyebuchi Chikezie, Ezeh Gloria Nwabugo, Agubor Cosmos Kemdirim, Nkwachukwu Chukwuchekwa}

Department of Electrical \& Electronic Engineering, Federal University of Technology, Owerri, Nigeria

\section{Email address:}

buchinosiri@gmail.com (Nosiri O. C.), ugoezeh2002@yahoo.com (Ezeh G. N.), aguborcosy@yahoo.com (Agubor C. K.), nonwuchekwa2002@yahoo.com (Chukwuchekwa N.)

\section{To cite this article:}

Nosiri Onyebuchi Chikezie, Ezeh Gloria Nwabugo, Agubor Cosmos Kemdirim, Nkwachukwu Chukwuchekwa. Efficient Sideband Noise Cancellation for Co-located Networks Using ANCT. American Journal of Networks and Communications. Vol. 4, No. 3, 2015 , pp. 75-84. doi: 10.11648/j.ajnc.20150403.19

\begin{abstract}
An efficient noise cancellation technique for a co-located network was realized using Adaptive Noise Cancellation Technique (ANCT). The technique was developed as improved feature to the classical application of passive filters. The paper focused on achieving a theoretical perfect cancellation considering three essential parameters: the amplitude, phase and delay characteristics of the signal and noise at both the primary path and the reference path of the co-located system. An experimental test-bed of the ANC Architecture was developed using Matlab-Simulink block design which demonstrated the error signals before and after cancellation.
\end{abstract}

Keywords: Co-location, Noise, Interference, Cancellation, Adaptive

\section{Introduction}

Wireless communication networks are faced with interference challenges which require urgent attention for improved system performance. Co-location or infrastructure sharing has been an aspect of interest in the fields of wireless communication. Government armed forces in the United States were the first to adopt the strategy. They required different wireless platforms to share a small site because of the mobile nature of their equipment (e.g. battleships, aircraft and expeditionary fighting vehicles $[1,2]$. The strategy was introduced to reduce the incessant proliferation of cell towers which are capital intensive and could pose environment health hazard. The policy was globally adopted as a reliable means of reducing capital and operating expenditure to promote economic efficiency and improves network performance deployment for operators in telecommunication industry [3]. Telecommunication infrastructure sharing is of two types, namely; passive and active components sharing [4]. Active sharing involves sharing of electronic components and facilities such as base station, microwave radio equipment, switches, antennas and receivers while passive sharing involves sharing of non-electronic components and facilities such as towers, shelters, electric supply, easements and ducts.
The infrastructure sharing technique considered in this article is passive component sharing.

In a co-located setting, base station receivers are required to receive weak desired signals in the presence of high-power transmit signals, resulting to major interference [5]. When RF signal is amplified to form the transmit signal, a significant amount of emissions are generated outside the transmit band referred to as sideband noise emission [6]. The emissions are due to the non-linearity and noise generated inside the power amplifier, and may appear as a "skirt" or "shoulders" when observed through the power spectrum at the output of power amplifier. These emissions or undesired noise energy may fall within the pass bands of a co-located receiver, degrading the receiver sensitivity and increasing the noise floor level. These undesired noise energy also contribute to the Carrier to Interference $(\mathrm{C} / \mathrm{I})$ ratio degradation, reduction in the full utilization of the capacity and the coverage radius, thereby disfranchising the end users from enjoying their hard paid services. Therefore, the undesired spectral components are required to be optimally reduced to avoid the introduction of excessive noise in the receiver front end.

Interference between the transmitted signals and the received signals depends on factors such as: The number of active channels at the site; the interval between the working 
frequency ranges of the two systems; the spatial separation between the receiver and transmitter and the characteristics of the technology including base station equipment [7].

This work predominantly considered the application of Adaptive Noise Cancellation Technique (ANCT) to filter co-located high power transmit jammers and allow only the desired receive signal at the receiver. The technique generally comes into play as a measure to improve on the limitations of other industrial interference mitigation techniques for co-located networks such as passive filters.

The technique also operates on the principle of destructive interference between the primary path and the reference path correlated noise signals. It can theoretically provide optimum cancellation for all distortion signals. It is inherently and unconditionally stable, requires no prior knowledge of the environment and possess better power handling capabilities with higher frequency agility response.

\subsection{Objective of the Study}

The paper was focused on developing an ANCT towards achieving theoretical perfect error cancellation. It also required developing an expression that will characterize the quality with which information is transferred through the channel and the minimum required information carrying characteristics of the signal such that the information can be properly detected and recovered.

\subsection{Significance of the Study}

The article contributes to providing improved cellular network performance in a worst case scenario involving a co-located network. These include: enhanced capacity and coverage performance, guaranteed optimum network availability and rapid expansion of customer base services.

\section{Literature Review}

The application of active cancellation technique for

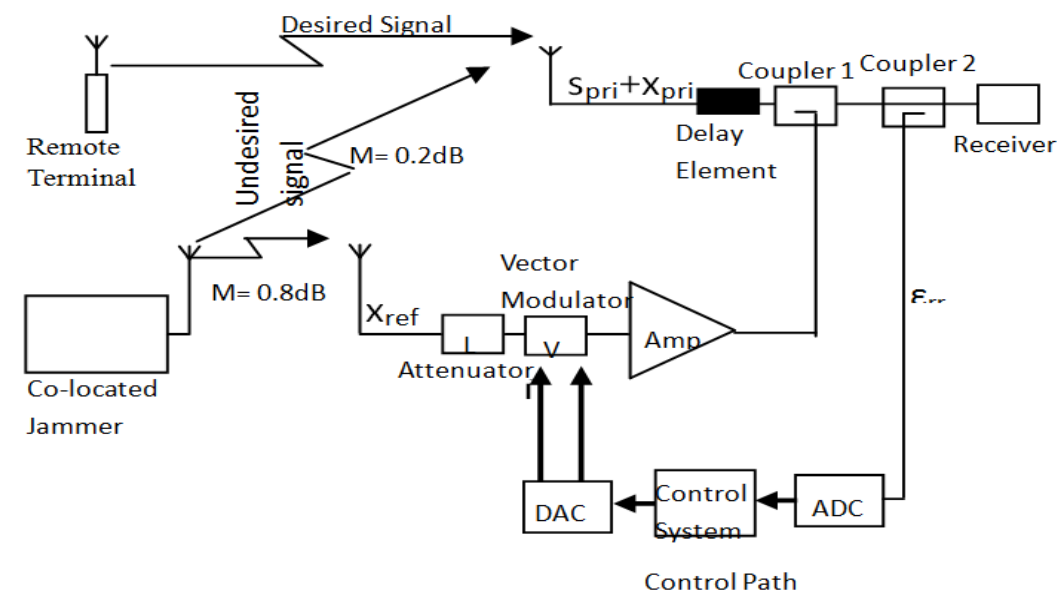

Figure 1. Conceptual Adaptive Cancellation System for a co-located.

The interfering signal was sampled using a reference antenna oriented in the direction of arrival of the interfering signal or directly from the source such as the output of the
Transmitter/Receiver (Tx/Rx) feed-through in auxiliary transmitters as developed by [8], demonstrated how a flexible multiband front end involving an additional multi-band transmitter chain was used to cancel the transmitter interference signal. The cancellation signal was computed over the Transmission channel bandwidth in the baseband and then transformed up to radio frequency via an auxiliary transmitter. The design does not cancel the transmitter noise in the receiver band because the transmitter noise and the auxiliary transmitter noise introduced are not correlated, instead of cancelling the transmitter noise, the technique introduced additional noise components to the receiver. Also, the introduction of auxiliary transmitter adds more cost to the design.

Single-loop adaptive cancellation system for co-locating a global positioning system (GPS) receiver unit within mobile communication user equipments was developed by [9] and [10]. The cancellation system coupled out a sample of the interfering mobile communication transmit signal. The compensation path signal was then adaptively gain-phase adjusted such that it is equal in magnitude and $180^{\circ}$ out of phase to the interference path signal when coupled back in the receive path of the GPS unit. This mitigates the interfering mobile communication transmit signal before it reaches the GPS receiver. A variable attenuator and a phase shifter can only cancel narrow-band noise signals. Accurate delay match for compensation path was deficient in the design.

\section{Methodology}

ANCT was applied as a technique to sample the undesired spectral components that interfere with the receiver front end. The primary antenna picks up the desired signal, $S_{\mathrm{Pr} i}$ with the jamming signals $X_{\operatorname{Pr} i}$. The reference antenna was directed to pick only the jamming signal $X_{\operatorname{Re} f}$, illustrated in Fig. 1 
receiver channel.

The ANCT signal controller consists of two components at the cancellation path: The vector modulator and amplifier. The vector modulator was necessary to modify the noise signal such that it matches its complement in the primary path. An attenuator in the vector modulator was required when the amplitude noise signals picked on the reference antenna was sufficiently larger than that on the primary. The amplifier was necessary to compensate for the insertion loss generated by the vector modulator (I and $\mathrm{Q}$ ) and provides the amplification needed to cancel the noise correlated signals at the primary path. The vector modulator works by adjusting the I and Q components of the noise signal. These modulators act as an actuator used to adjust the cancellation signal in the cancellation signal path, usually described as the weight variables of the adaptive systems. The reference path and the primary path have different delay characteristics; the reason was that each path uses different components. The primary path consists of two directional couplers and a delay line, exhibit fairly active component characteristics with low insertion loss while the reference path contains both passive and active RF components, namely attenuator, vector modulator and amplifier.

The ANCT was implemented using MATLAB-Simulink test bed as shown in Fig. 2. Let the signal generator A, generates the desired signal and represented as

$$
S_{G A}(t)=V_{S p r i} \sin \left(2 \pi f_{c} t+\varphi_{S p r i}\right)
$$

where $S_{G A}=S_{p r i}$,

- $f_{c}$ is the instantaneous frequency,

- $\varphi_{\text {Spri }}$ is the initial phase of the signal,

- $V_{S p r i}$ is the amplitude of the desired signal at the primary path.

Let the signal generator B generates the interfering signal and represented as

$$
C_{A}(t)=X_{\mathrm{Pr} i}(t)+S_{\mathrm{Pr} i}(t)=V_{X \mathrm{Pr} i} \cos \left(2 \pi f_{c} t-\beta c I_{X \mathrm{P} r i}+\varphi_{X \mathrm{P} \mathrm{r} i}\right)+V_{S \operatorname{Pr} i} \sin \left(2 \pi f_{c} t+\varphi_{S \mathrm{Pr} i}\right)
$$

At the input of the Vector Modulator the interfering signal was represented as:

$$
X_{\operatorname{Re} f}(t)=V_{X \operatorname{Re} f} \cos \left(2 \pi f_{c} t-\beta c I_{X \operatorname{Re} f}+\varphi_{X \operatorname{Re} f}\right)
$$

The expected output of the vector modulator is

$$
X_{0 \operatorname{Re} f}(t)=V_{X \operatorname{Re} f}(t) \cos \left\{2 \pi f_{c} t-\beta c I_{X \operatorname{Re} f}+\left(\varphi_{X \operatorname{Re} f}+180^{\circ}\right)\right\}
$$

At the Coupler 1, which is the summation point or cancellation point?

$$
X_{R}=X_{\operatorname{Re} f}-X_{\operatorname{Pr} i}=e_{r r}
$$

Where $X_{R}$ is the residual noise signal .

The magnitude and phase characteristics of the error signal depends on the phase error, amplitude imbalance and delay mismatch between the primary signal path and reference signal path at the point of cancellation.
Let $\Delta_{V}$ represent the difference in amplitude imbalance

$\theta_{e r r}$ represent the phase error and

$\Delta_{L}$ represent the delay mismatch

$$
\begin{gathered}
\Delta_{V}=V_{X \operatorname{Re} f}-V_{X \operatorname{Pr} i}, \\
\therefore V_{X \operatorname{Re} f}=\Delta_{V}+V_{X \operatorname{Pr} i} \\
\theta_{e r r}=\left(\varphi_{X \operatorname{Re} f}-\varphi_{X \operatorname{Pr} i}\right)-180^{0},
\end{gathered}
$$




$$
\begin{aligned}
\therefore \varphi_{X \mathrm{Re} f} & =\theta_{e r r}+\varphi_{X \mathrm{Pr} i}+180^{\circ} \\
\Delta_{L} & =I_{X \mathrm{Re} f}-I_{X \mathrm{Pr} i},
\end{aligned}
$$

$$
X_{\mathrm{Re} f}=\left(\Delta_{V}+V_{X \mathrm{Pr} i}\right) \cos \left(2 \pi f_{c}-\beta c\left(\Delta_{L}+I_{X \mathrm{P} r i}\right)+\theta_{e r r}+\varphi_{X \mathrm{P} \mathrm{r} i}+180^{\circ}\right.
$$

Hence, $\varepsilon_{r r}$ is the outcome of the vector addition of the primary signal and the reference signal at the cancellation point, which takes place at coupler B.

$$
\therefore \varepsilon_{r r}=\varepsilon_{X r e f}+\varepsilon_{X \operatorname{Pr} i}
$$

\section{Signal}
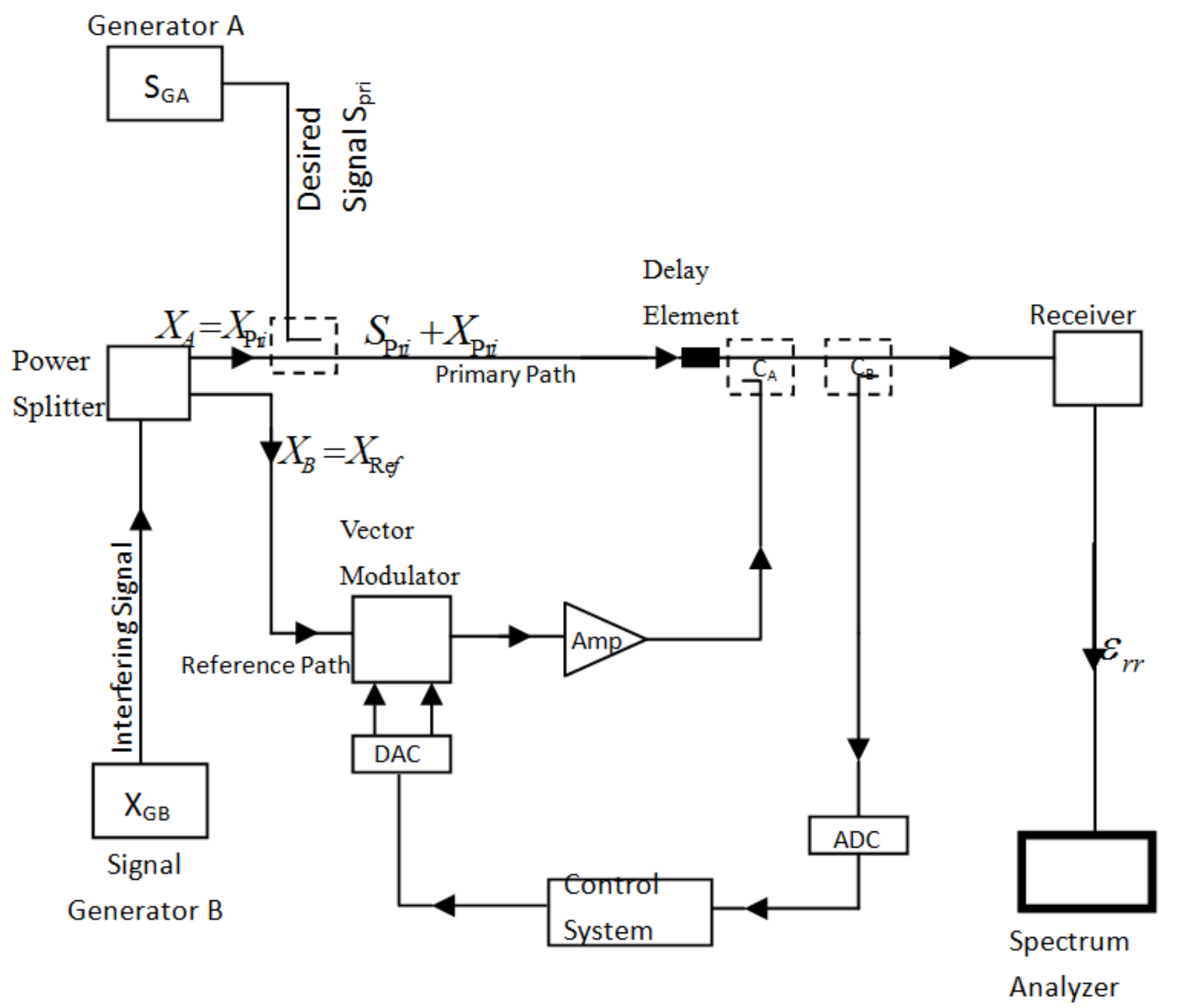

Figure 2. Implementation Test-Bed of the ANCT. 


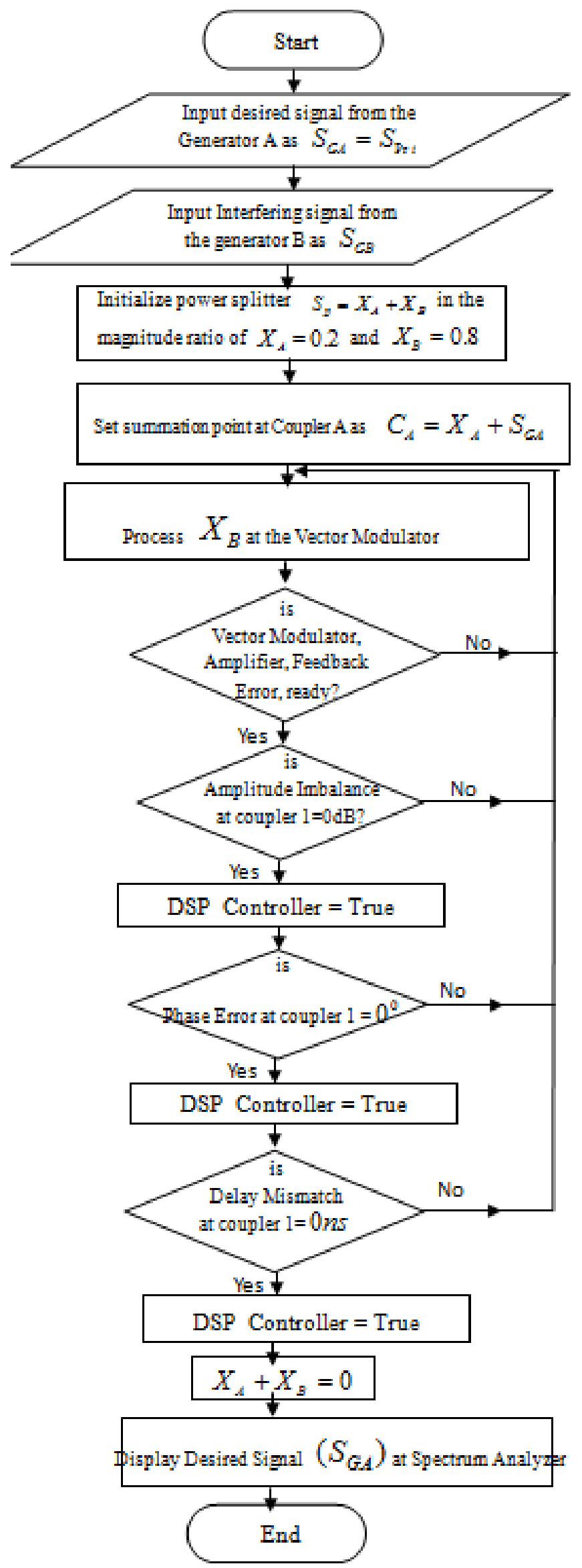

Figure 3. Steps for implementing the ANC system represented in a flowchart algorithm. 


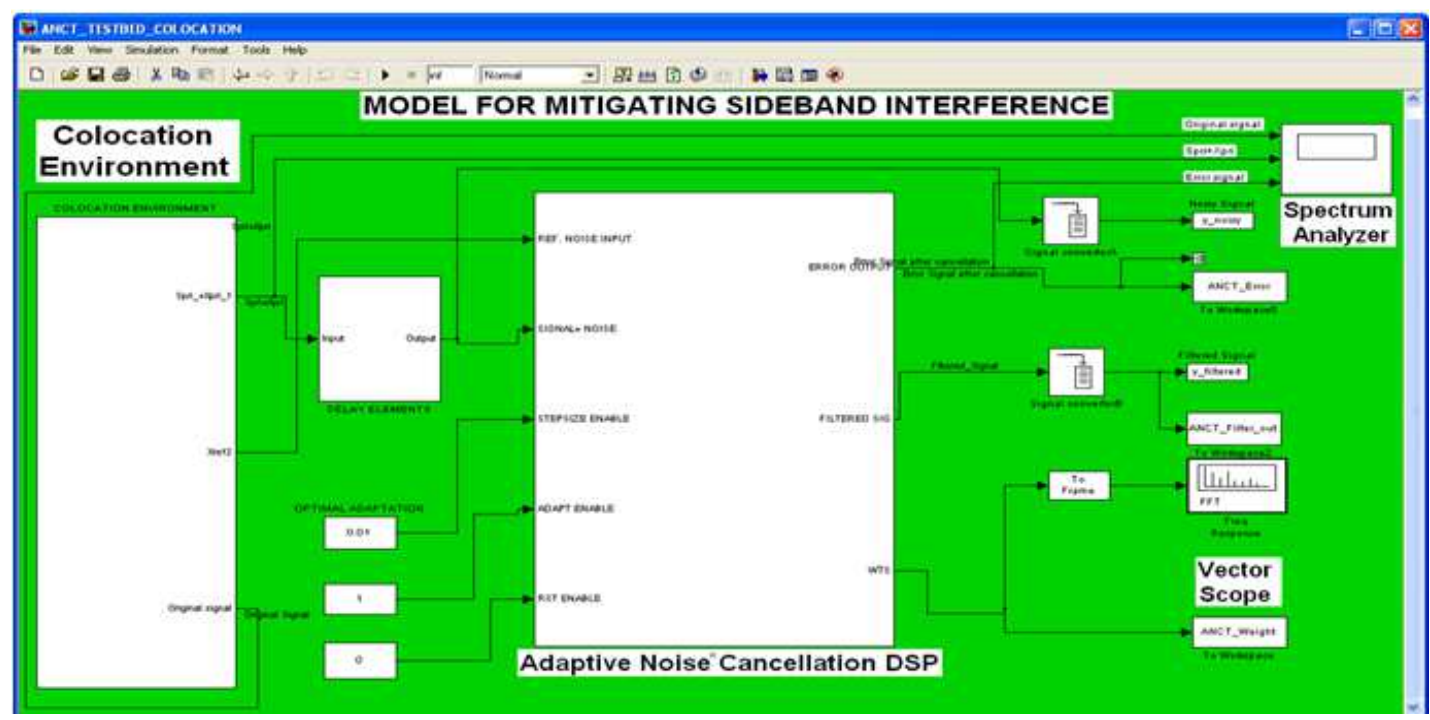

Figure 4. Matlab-Simulink Test-bed for the Adaptive Noise Cancellation System.

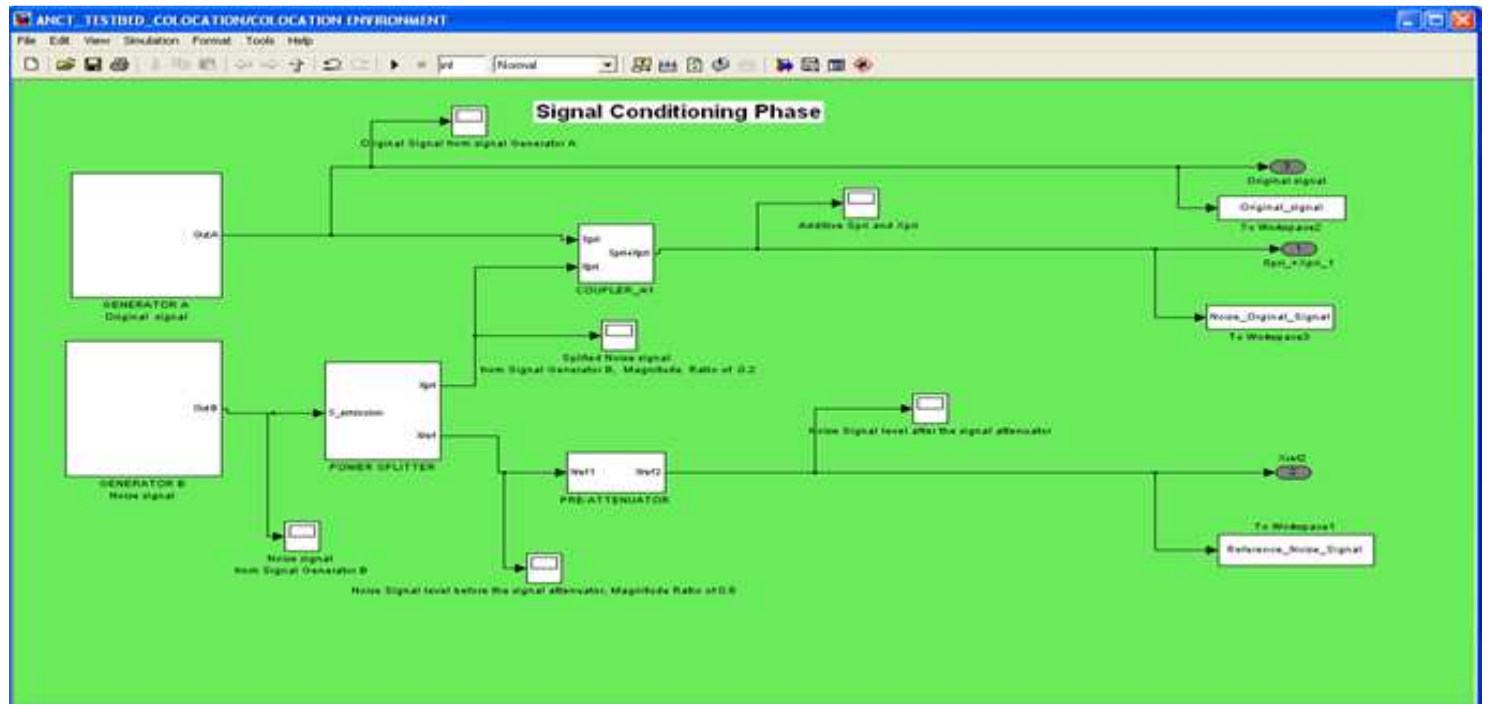

Figure 5. Signal Conditioning Phase.

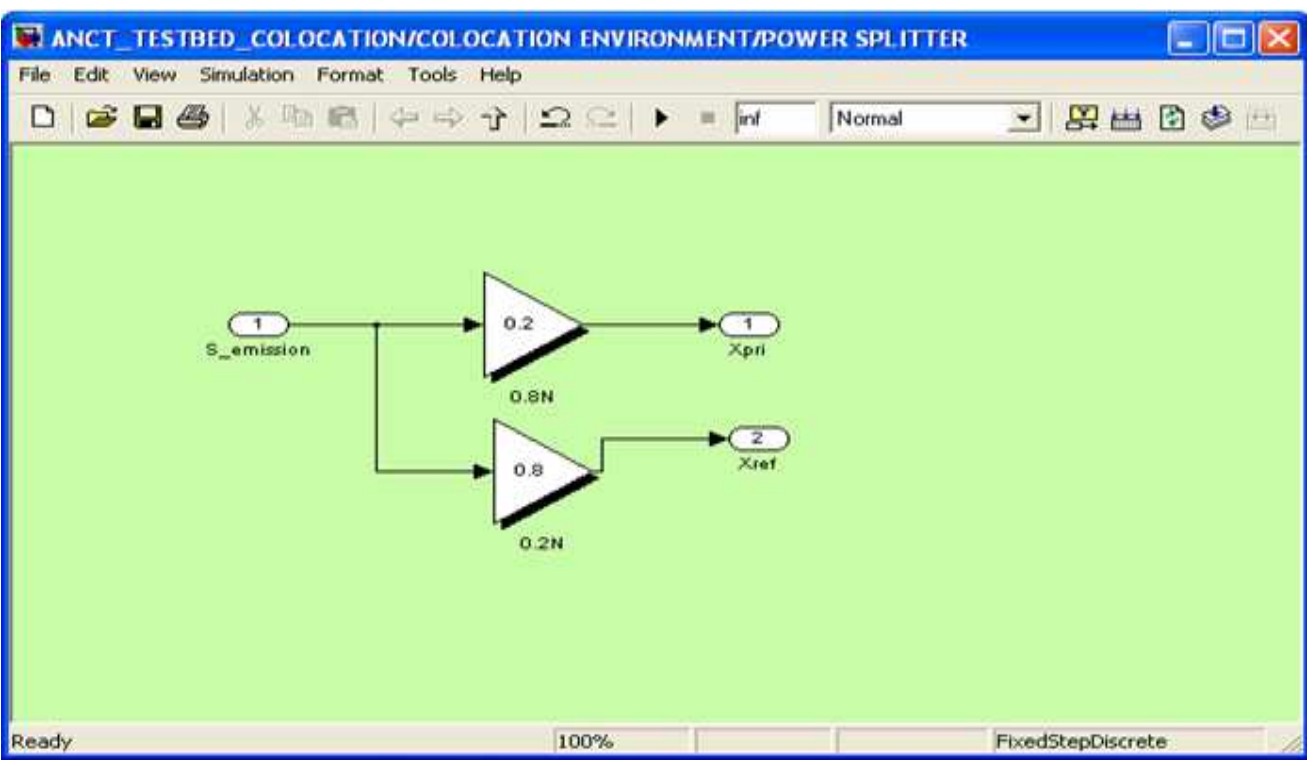

Figure 6. Power Splitter. 


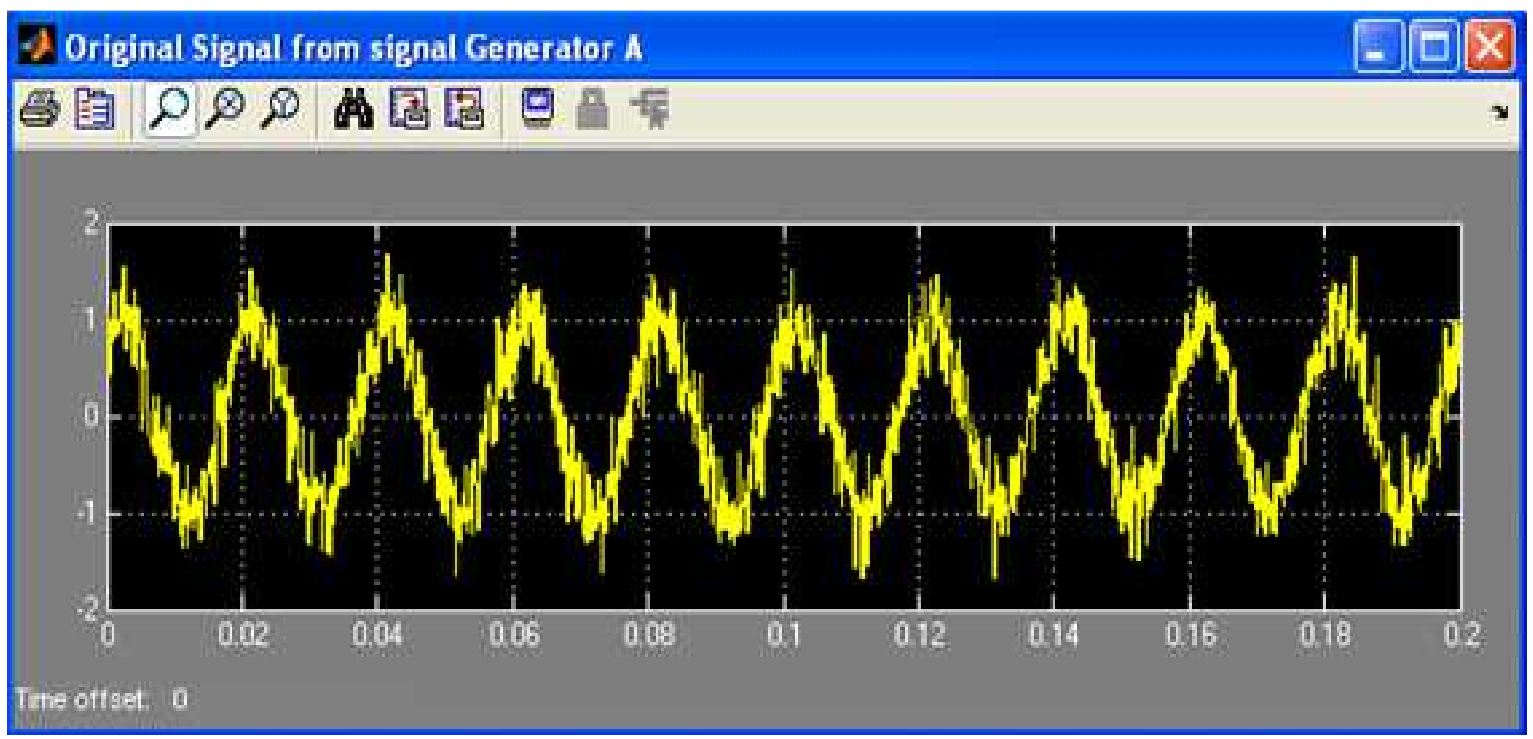

Figure 7. Original Signal from Signal Generator A.

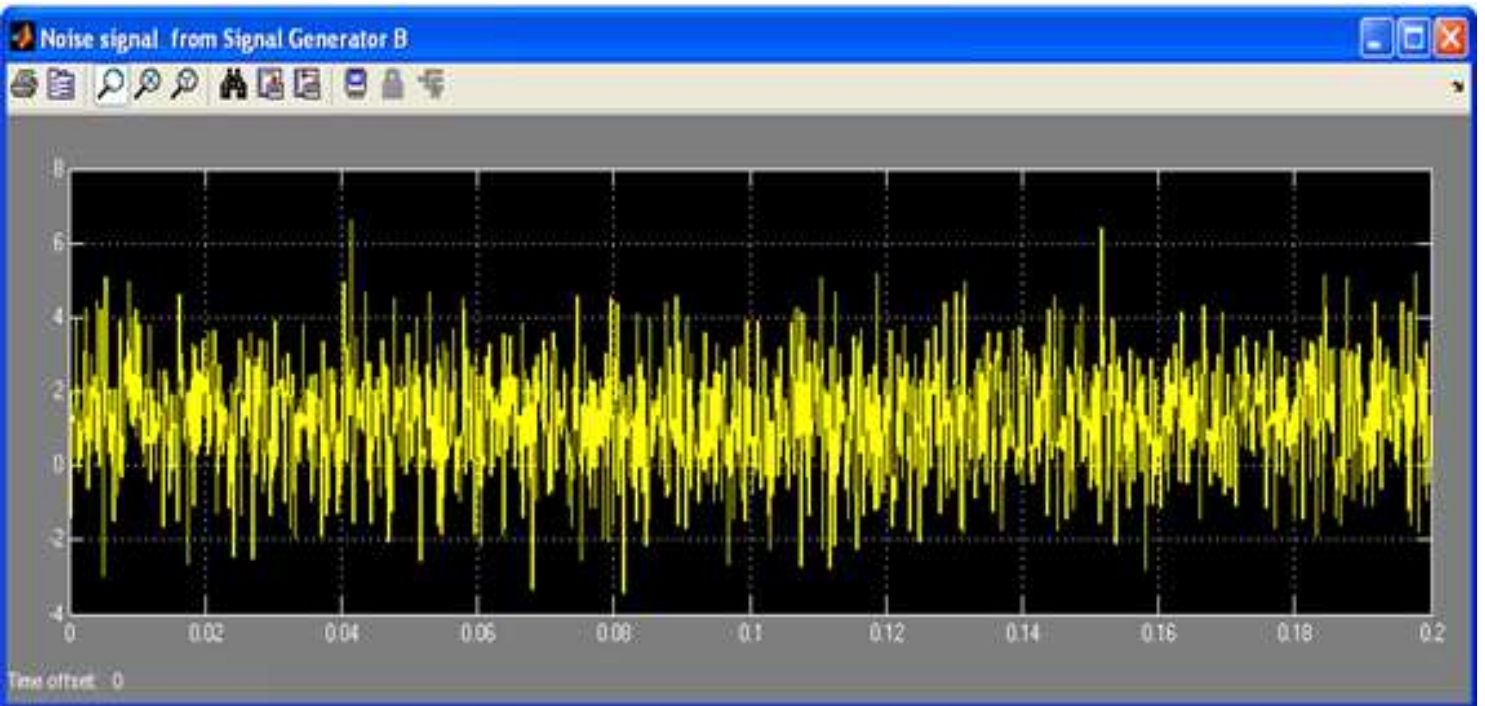

Figure 8. Noise Signal from Signal Generator B.

A Splited Noise signal from Signal Generator B, Magnitude Ratio of 0.2 -

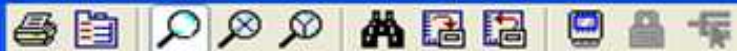

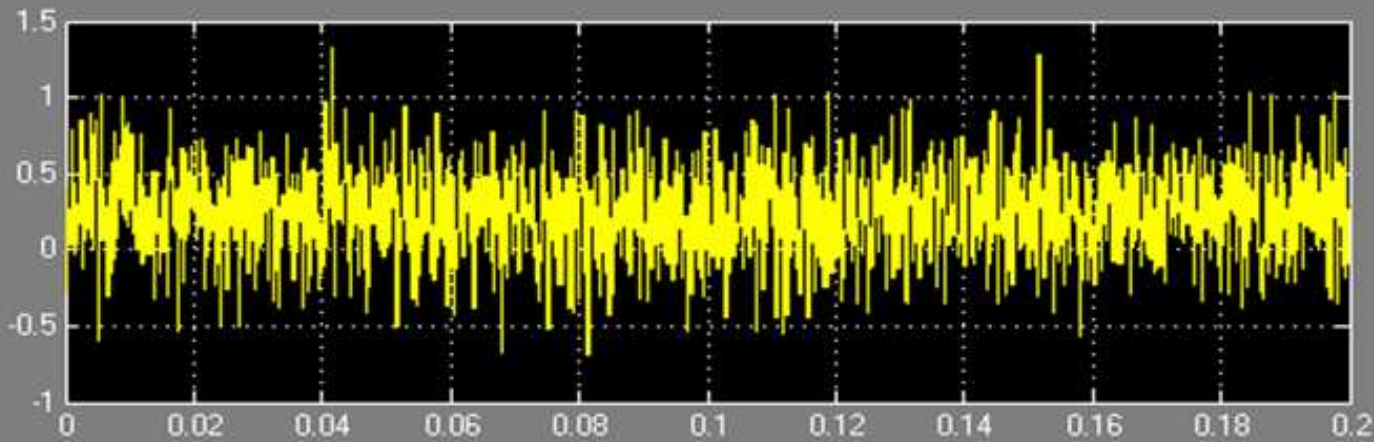

Time offset: 0

Figure 9. Splitted Noise signal from Signal Generator B, Magnitude Ratio 0.2. 


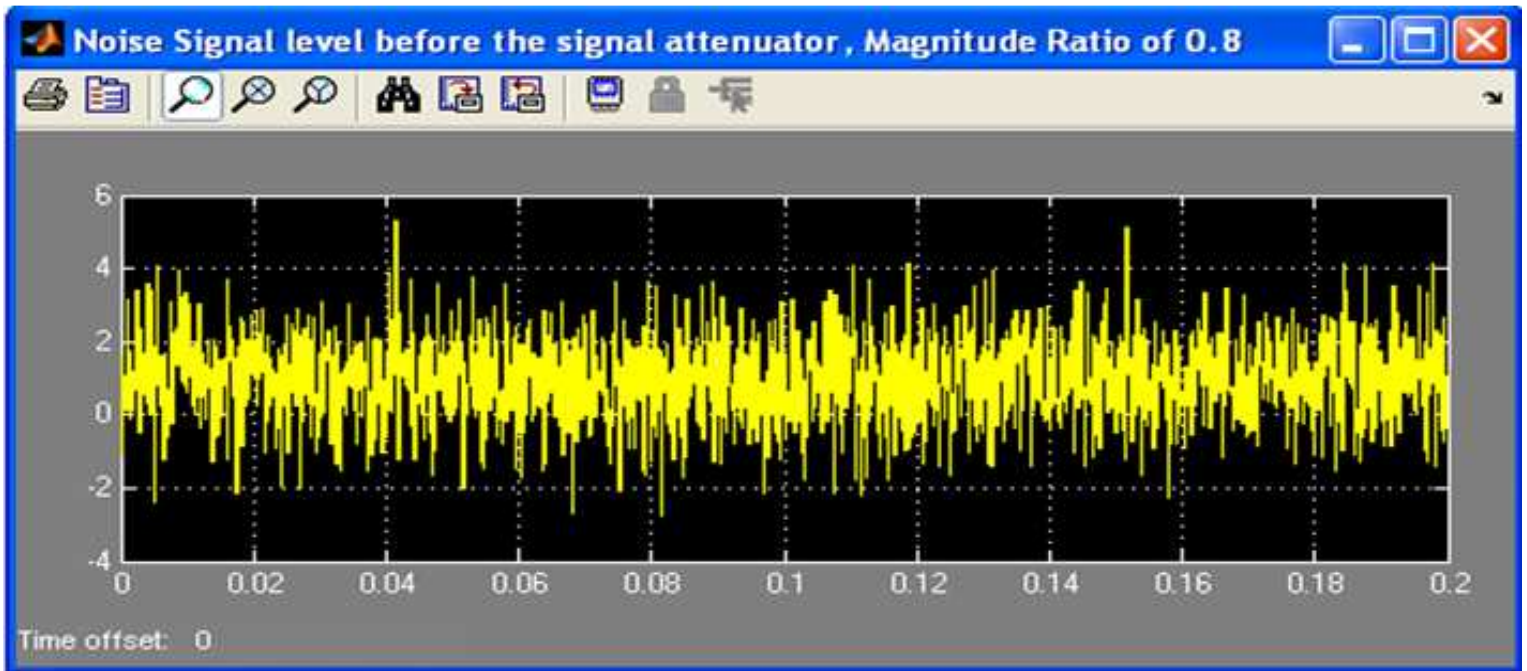

Figure 10. Noise signal level before the signal attenuator, Magnitude Ratio 0.8.

Noise Signal level after the signal attenuator

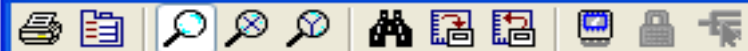

$\square \square$

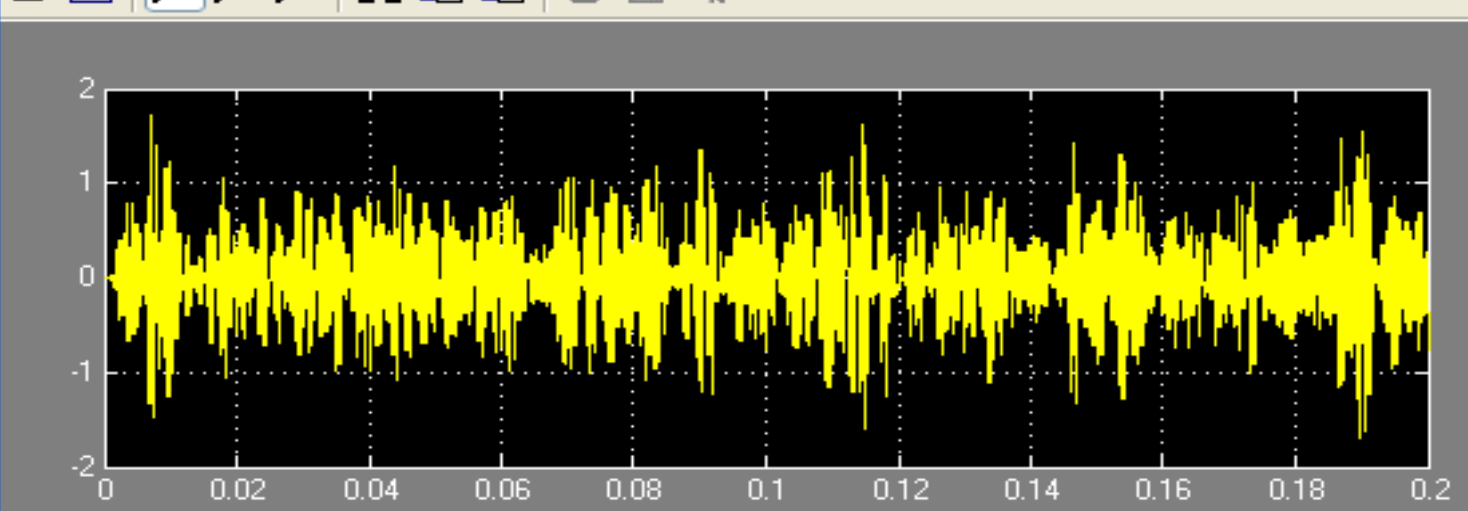

Time offset: 0

Figure 11. Noise signal level after the signal attenuator.

Additive Spri and Xpri

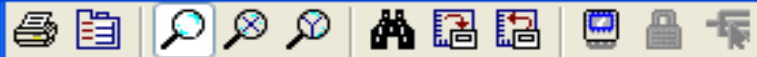

$\square \square$

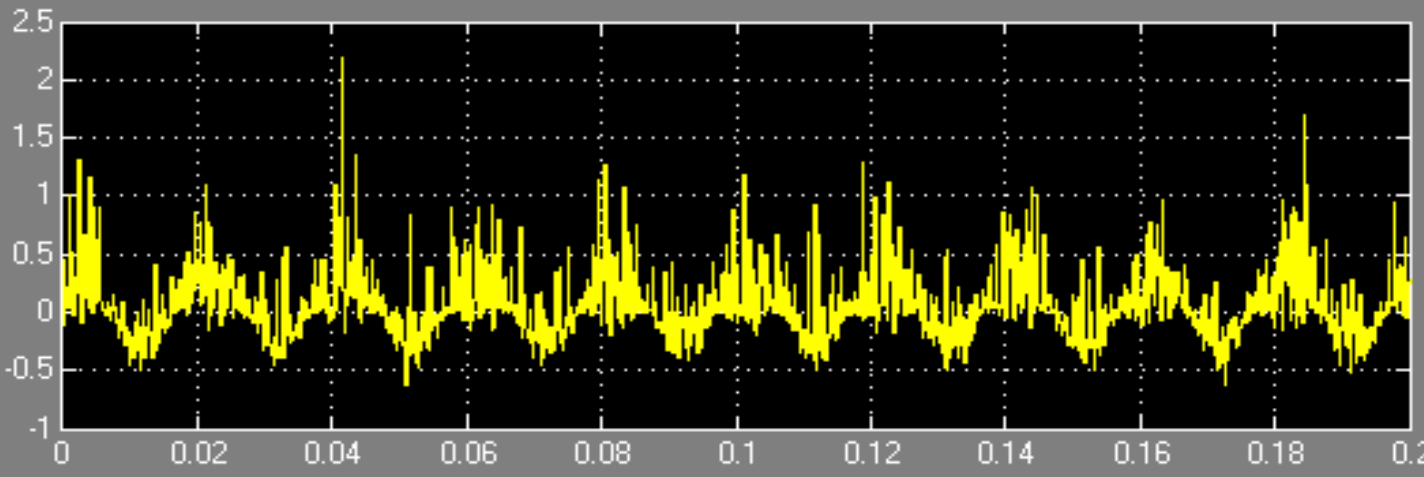

Time offset: 0

Figure 12. Additive Spri and Xpri. 


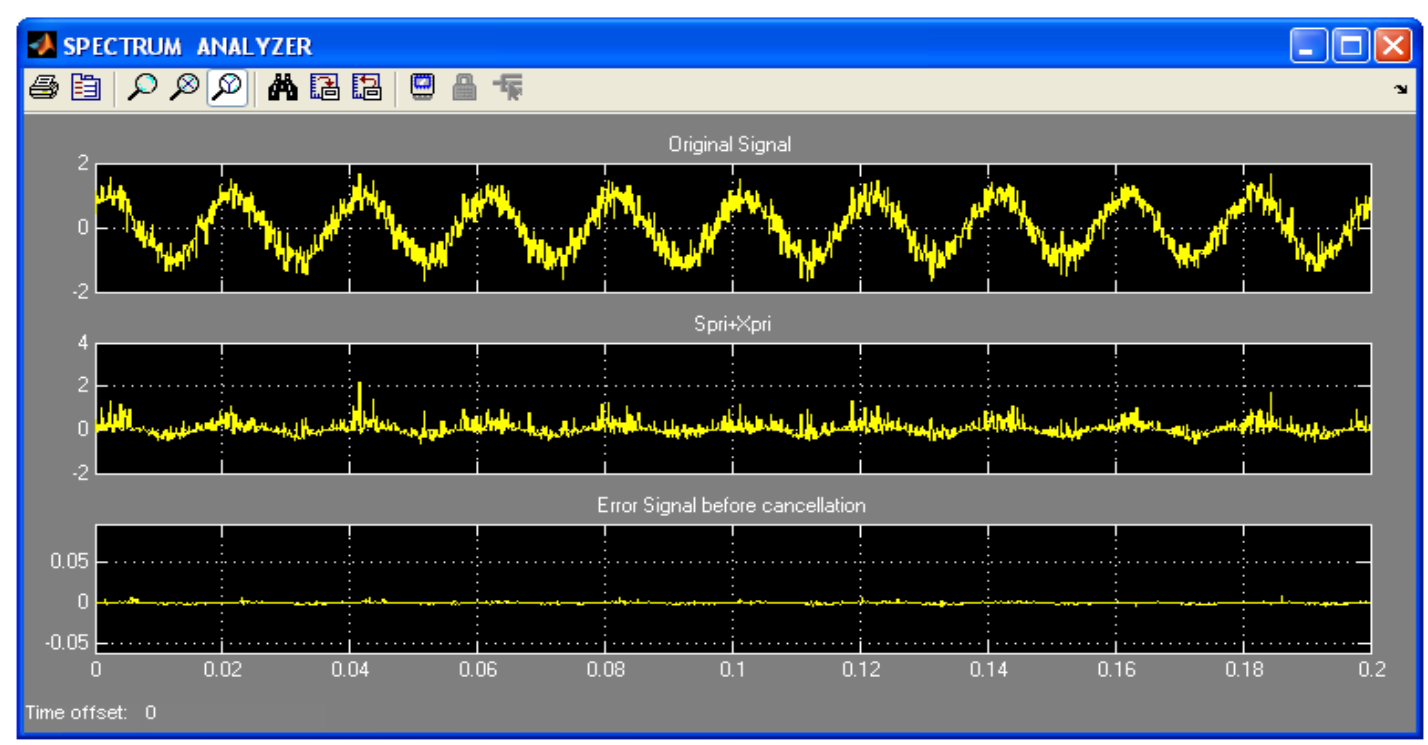

Figure 13. Error Signal before cancellation.

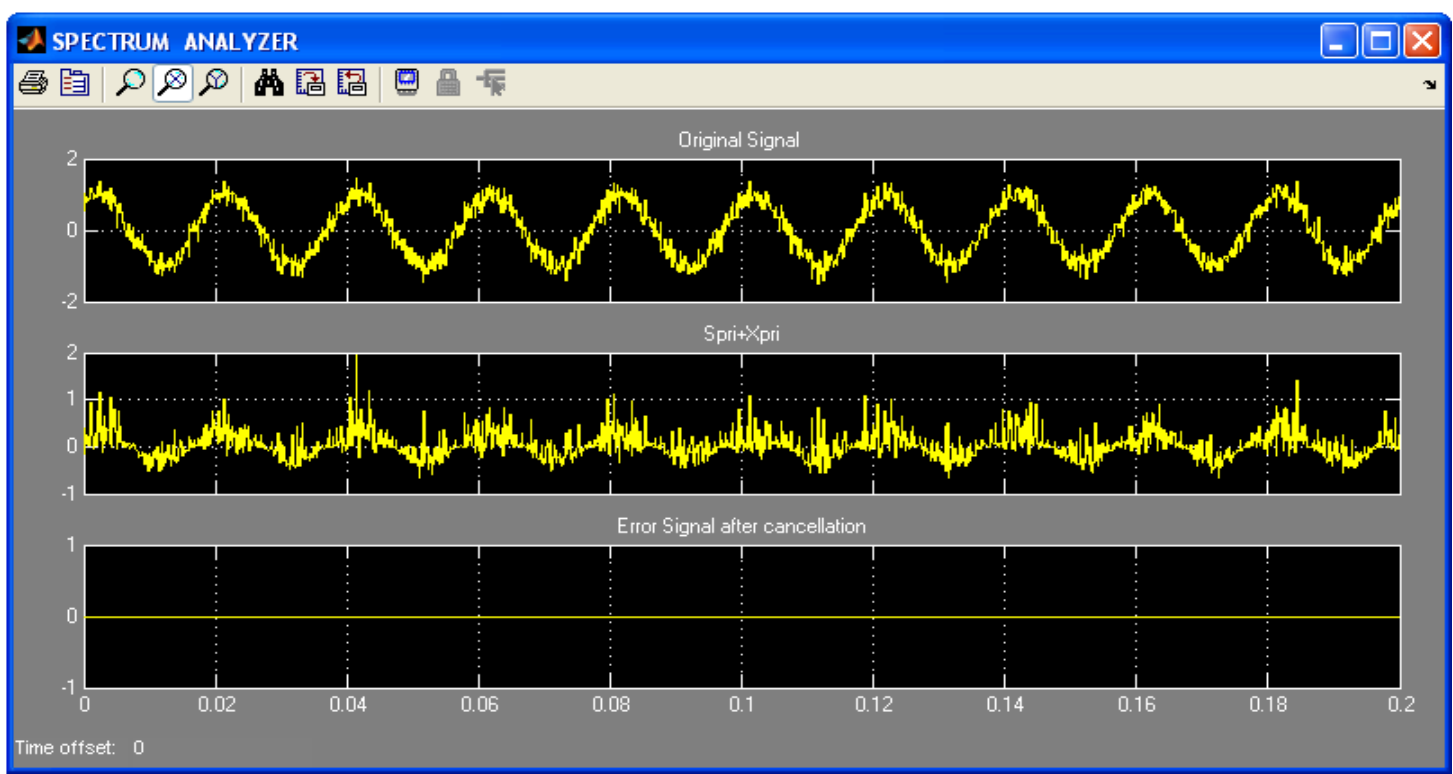

Figure 14. Error Signal after cancellation.

\section{Conclusion}

Adaptive Noise Cancellation Technique was once a weapon against intentional jammers, now a weapon against unintentional jammers. It has been shown capable of eliminating in-band interference over a wider bandwidth and over a changing environment without appreciably affecting the desired signal. The study was primarily aimed at providing an improved solution to mitigate sideband noise in a co-located network compare to the classical passive filters. The technique was primarily focused on achieving perfect theoretical cancellation where the amplitude imbalance, phase error and delay mismatch are dynamically adjusted by the weight variables towards achieving perfect cancellation. An experimental test-bed set-up was performed using Matlab-simulink environment to realize the error cancellation performances before and after cancellation, implemented using a developed algorithm.

\section{References}

[1] German F., Annamalai K., Young M., Miller M.C., "Simulations and Data Management for Co-site Interference Prediction", in Proc. IEEE International symposium on Electromagnetic compatibility, July 2010.

[2] Demirkiran I., Weiner D.D., Drozd A. and Kasperovich I., "Knowledge-based Approach to Interference Mitigation for EMC of Transceivers on Unmanned Aircraft," in Proc. IEEE International Symposium on Electromagnetic Compatibility, July, 2010.

[3] Louay A. C., Bahjat E., Ghassan H., Mohamad M., "Telecom Infrastructure Sharing: Regulatory Enablers and Economic Benefits," Booz Allen Hamilton, 2007. 
[4] Bala-Gbogbo E., "Telecom Industry Operators Opt for Infrastructure Sharing.", 2009.

[5] Razavi B., "RF Microelectronics". Upper Saddle River, NJ: Prentice Hall, 1998,pp. 11-53.106.

[6] A. Roussel "Feedforward interference cancellation system applied to the $800 \mathrm{MHz}$ CDMA cellular band". A Master of applied science in Electrical Engineering. Ottawa-Carleton Institute. $\operatorname{Pg} 6,2003$.

[7] Infrastructure sharing and collocation services License by Nigerian Communications Commission Section 32 of the Nigerian Communications Act, 2003.
[8] Charu S. and Monika B. "Active cancellation concept of the $\mathrm{Tx} / \mathrm{Rx}$ feedthrough applying an auxiliary transmitter", International Journal of Computer science Issues, 2011

[9] Thomas H., Kodim W., Gloeckler R., Dingfelder H., "Transmitter Leakage Cancellation Circuit for Co-Located GPS Receiver," European Patent 1 091497, 11 April 2001.

[10] McConnell R. J., Tso R., "Method and Apparatus for Reducing GPS Receiver Jamming during Transmission in a Wireless Receiver," U.S. Patent 6961 019, 1 Nov 2005. 